\title{
Acute perilymphatic perfusion of the guinea pig cochlea
}

\author{
Alfred L. Nuttall, Michael J. LaRouere and Merle Lawrence \\ Department of Otorhinolaryngology, Kresge Hearing Research Institute, University of Michigan Medical \\ School, 1301 E. Ann Street, Ann Arbor, MI 48109, U.S.A.
}

(Received 10 April 1981; accepted 17 September 1981)

\begin{abstract}
A method for the continuous perfusion of the perilymphatic space of the inner ear in the guinea pig is described. Artificial perilymph is supplied to the cochlea and drained away through a tubing system while flow rates from $10 \mu 1 / \mathrm{min}$ to $0.3 \mathrm{ml} / \mathrm{min}$ are established by gravity syphon pressure. Techniques are also presented which allow control over the temperature of the perfusate and over the level of dissolved oxygen in the perfusate. Along with these variables, the $\mathrm{pH}$ of the artificial perilymph can be manipulated and various drugs can be added to the perfusate to test their effect on the inner ear. The function of the inner ear is monitored by continuous recording of the sound evoked bioelectric potentials, the cochlear microphonic and the compound action potential. The cochlear perfusion technique has many applications in the study of cochlear physiology and metabolism, and in testing the sensitivity of the inner ear to ototoxic drugs.
\end{abstract}

Key words: perfusion; perilymph; cochlea; guinea pig; aminoglycoside antibiotics; cochlear temperature; perilymph $\mathrm{pH}$; intracochlear oxygen tension.

\section{Introduction}

There are many types of studies on the inner ear in which the cochlear fluids have specific importance. These studies can range from the mechanical properties of the fluids (as related to wave motion within the cochlea) to their chemical constituency and their role in the transport of metabolic components to the sensory epithelium. Any technique which provides a means of controlling cochlear fluids is useful to the above studies. For example, if the concentration of a drug, which alters inner ear function, such as an aminoglycoside antibiotic, is known within the cochlear fluids, then the activity of the drug is better understood than if the drug concentration is known only for blood plasma. This is because of the possible blood-cochlear fluid barriers.

Perilymphatic perfusion is a method which allows the control of fluid parameters throughout the perilymphatic space of the cochlea. The perfusion is continuous, flowing at a sufficiently high rate such that drug concentrations are constant, uninfluenced by clearance or metabolism. In effect, the method provides a means for topical application of chemical agents into the sensory epithelium. During perfusion, 
sound stimuli can then be applied to the inner ear via an intact middle ear mechanism. Sound induced bioelectric signals from the cochlea and/or other auditory structures, are available for analysis. These signals are measures of auditory function which can indicate the effects, on the inner ear, caused by altering the perilymphatic environment.

Perilymphatic perfusion is not a new technique. One of the first uses of inner ear perfusion was in a study by Tasaki and Fernandez [10]. Since then several other investigators have used perfusion in one form or another (e.g. [1]). Nor is perilymphatic perfusion the only form of cochlear perfusion, as it is also possible to perfuse the endolymphatic space of the cochlea (e.g. [3]). However, the previous uses of perilymphatic perfusion have not exhausted the promise that this technique holds for new studies of the auditory receptor organ and they have not adequately addressed the important methodological variables of perfusate $\mathrm{pH}$, temperature, and dissolved oxygen concentration.

The purpose of this report is to provide methodological details for perilymphatic perfusion of the cochlea. A number of critical control studies are presented along with example results from studies of aminoglycoside drug ototoxicity, where the perfusion technique was utilized. At its present stage of development in this laboratory, the method is a routine procedure which has high yield of successful experimental animals. The level of control over experimental variables greatly exceeds that of the earlier reports and some important methodological problems are resolved.

\section{Methods}

Albino and pigmented quinea pigs with body weights between 200 and $500 \mathrm{~g}$ have been used in the perfusion studies. The animals were anesthetized with Dial in Urethane anesthetic $(0.8 \mathrm{ml} / \mathrm{kg}$ i.p.) and artificially respirated. A muscle relaxant (d-tubocurarine chloride $0.3 \mathrm{mg}$ i.m.) was administered to prevent middle ear muscle contractions and body movement which could disturb the connection of the perfusion tubes. Heart rate was monitored and supplemental heat was provided to the torso to maintain normal rectal temperature $\left(38.5^{\circ} \mathrm{C}\right)$.

A ventro-postauricular surgical dissection was carried out to expose the auditory bulla. The head was then securely fastened in a headholder which allowed access to the ventral side of the head and neck.

The ventral portion of the bulla was opened carefully with preservation of the tympanic membrane and middle ear ossicles. After the mucosal lining over the bony cochlea was brushed away using a cotton pledget, two holes were drilled into the first or basal-most turn of the cochlea. One hole was into the scala tympani space while the other was into the scala vestibuli. The positions of the holes are indicated schematically in Fig. 1, and in the histological sections shown in Fig. 2. The holes were drilled using a hand-held drill which was constructed by sharpening the bit of a jeweler's screwdriver. The pointed screwdriver bit and its handle function extremely well for this purpose and circular holes as small as $25 \mu \mathrm{m}$ can be created in the 


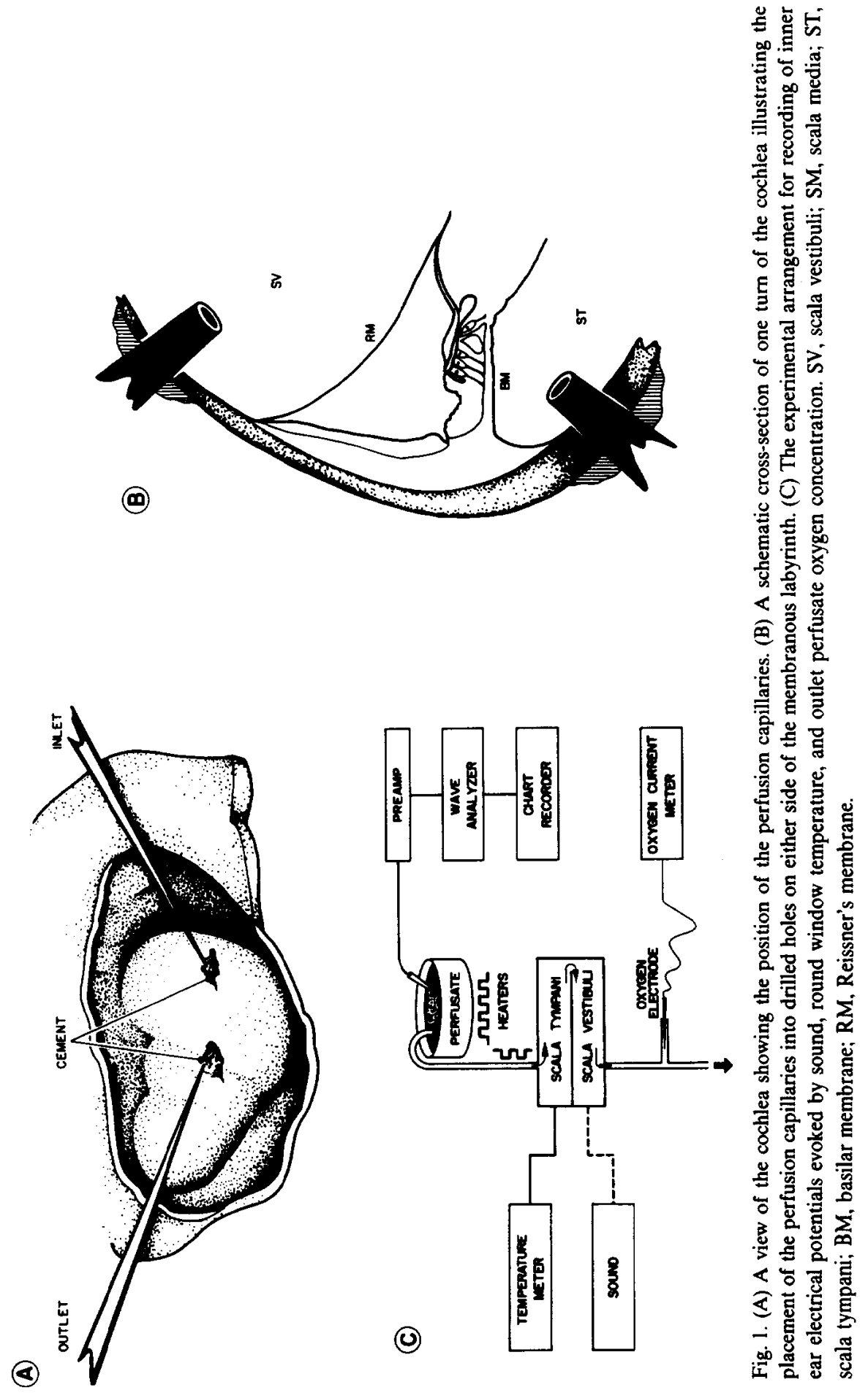




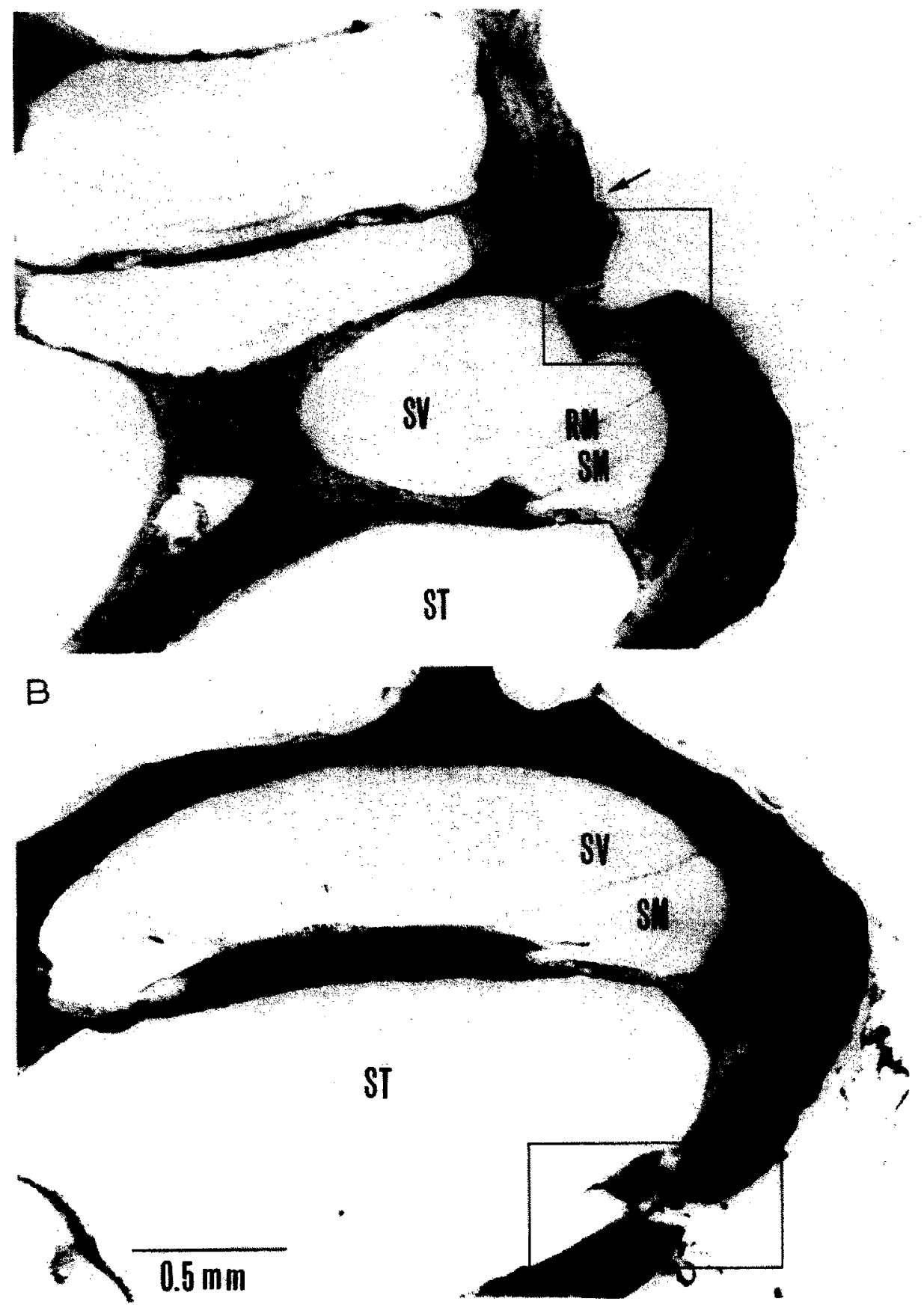

Fig. 2. Histological sections of the guinea pig cochlea showing the actual positions and sizes of the perfusion openings. (A) The scala vestibuli opening (outlined). (B) The scala tympani opening (outlined). RM, Reissner's membrane; SV, scala vestibuli; ST, scala tympani; SM, scala media. Arrow points to a bony crease demarking the juncture of the first and second cochlear turns. 
cochlear wall. For the purpose of perfusion these holes are approximately $0.1-0.2$ $\mathrm{mm}$ in diameter.

The exact placement and size of the opening into the scala tympani in the first turn is not critical. There is a large space in this area of the cochlea (Fig. 2B) which ensures that a larger hole will not compromise important structures. The depth to which the drill bit or the perfusion capillary penetrates into the scala can be greater than $0.5 \mathrm{~mm}$. When drilling, it is necessary to avoid disturbing the spiral ligament and it is desirable to avoid rupturing blood vessels within the bony wall. This can be accomplished by appropriate back lighting of the cochlea which can allow observation of the blood vessels within the bony wall of the cochlea.

The position of the hole into the scala vestibuli is more critical and there is the possibility of damaging the spiral ligament near the attachment of Reissner's (vestibular) membrane. A histological section of the cochlea in Fig. 2A illustrates the problem. The position and angle of the drill must be such that a hole results in the apical-most third of the visible arch that represents the anterior directed surface of the first turn of the cochlea. The easiest landmark to use is the crease in the bony surface of the cochlea which segments the first turn from the second. The hole is drilled and angled posteriorly as close to the crease as possible.

As an aid to establishing a knowledge of the proper placement of the scala vestibuli opening, it is useful to obtain histological sections of the entire cochlea in the apical-basal plane. A ruptured vestibular membrane, which would be evident in these sections, would lead to a degradation of the cochlear potentials with time as a result of mixing of perilymph and endolymph [5].

The perfusion apparatus that connects to the cochlea consisted of an inlet and an outlet tubing system. Polyethylene (Intramedic PE60), Tygon $(0.9 \mathrm{~mm}$ i.d., $2.3 \mathrm{~mm}$ o.d.), or glass $(0.5 \mathrm{~mm}$ i.d., $0.8 \mathrm{~mm}$ o.d.) tubing was attached onto the end of a glass capillary tube (length $5 \mathrm{~cm}, 0.5 \mathrm{~mm}$ i.d., $0.8 \mathrm{~mm}$ o.d.) after the other end of the glass capillary was drawn out on an electrode puller. The drawn end creates a tip which was much smaller than the cochlear holes and it was sized by breaking the tip until it nearly fills the drilled hole. Each capillary and tubing was prefilled with artificial perilymph *, held by a micromanipulator and positioned into a hole.

In order to allow cement to seal around the capillaries and create a leak-free system, the natural efflux of fluid (perilymph plus cerebrospinal fluid) was absorbed by a cotton pledget placed near, but not in, the scala vestibuli hole. Durelon ${ }^{\text {(a }}$ (carboxylate) dental cement serves very well for cementing the capillary tube to the cochlea.

It is helpful to leave the syringes, used for prefilling the tubing and capillaries with perilymph, affixed to the tubing until the cement is dry. This will ensure the integrity of the fluid columns. Every effort must be made to avoid air bubbles in the tubing which would result in air lodging in the cochlea.

Flow was established by removing the prefilling syringes and raising the outlet tube level higher than the inlet in order to cause back flow and clear the inlet tubing

* Artificial perilymph had the following composition: $130 \mathrm{mM} \mathrm{NaCl} / 10 \mathrm{mM} \mathrm{NaHCO} / 4 \mathrm{mM}$ $\mathrm{KCl} / 1 \mathrm{mM} \mathrm{Mg} \mathrm{Cl} 2 / 1 \mathrm{mM} \mathrm{NaH} \mathrm{PO}_{4} / 20 \mathrm{mM}$ HEPES, $\mathrm{pH} 7.4$. 
of any bubbles formed after removal of the syringe needle. The inlet tube (scala tympani side) was placed in a container of artificial perilymph which served as a supply reservoir. The outlet tubing was then lowered below the level of the perilymph in the supply reservoir establishing flow which proceeds by entering the scala tympani and leaving the scala vestibuli. This scheme is detailed in Fig. 1A.

The length of the inlet-side tubing was chosen as $25 \mathrm{~cm}$ while the outlet was 75 $\mathrm{cm}$ long. The reasons for selecting these lengths were to minimize the time required to move a sample to the inner ear (short inlet) and to allow enough length on the outlet to select a wide range of fluid column heights to adjust flow rate (long outlet).

Flow of artificial perilymph was therefore governed by gravity syphon pressure and the scheme resulted in a constant pressure across the cochlea. Flow volume was usually set at $10-20 \mu 1 / \mathrm{min}$ although rates up to $0.3 \mathrm{ml} / \mathrm{min}$ have been successfully used.

Experiments requiring the control of $\mathrm{pH}$ were accomplished by manipulating the combination of the three buffers; Tris, malic acid, and HEPES, always keeping osmolality constant. A pH range from 6.9 to 8.5 was utilized. All other experiments utilized HEPES buffer artificial perilymph adjusted to $\mathrm{pH}$ 7.4-7.8.

When temperature was studied the perilymph was raised to the test temperature $\left(35-40^{\circ} \mathrm{C}\right)$ and held at that temperature until and during use. A heater coil was incorporated into the inlet delivery system (Fig. $1 \mathrm{C}$ and $3 \mathrm{~A}$ ) so that direct current could be applied resulting in control of the perfusate temperature, and consequently of cochlear temperature. A Chromel-Alumel (K-type) thermocouple, constructed from $0.075 \mathrm{~mm}$ wire was placed on the round window membrane of the cochlea to monitor the cochlear (perilymph) temperature. All other experiments were performed with room temperature $\left(21^{\circ} \mathrm{C}\right)$ perilymph.

Aminoglycoside antibiotics in different molar concentrations were dissolved in artificial perilymph and substituted for the control perilymph in order to test the effect of topical application of these drugs and to learn more about the relative toxicity of the different antibiotic compounds.

Dissolved oxygen in the artificial perilymph was specifically controlled in some experiments to determine if the perfusion technique is capable of gaining a metabolic control over inner ear oxygen availability. Oxygen concentration in the perilymph was altered by a $10 \mathrm{~min}$ bubbling of $\mathrm{O}_{2}$ or $\mathrm{N}_{2}$ (of known concentration) using a frit-type gas-washing container. Oxygen concentration in the artificial perilymph was measured by a polarographic technique with a Transidyne General Corp. oxygen meter and platinum-iridium wire microelectrodes (Frederic Haer Co.). Oxygen concentration changes in the outflowing artificial perilymph were measured with an oxygen-sensitive electrode which was incorporated into the outlet perfusion capillary system (Fig. 3C).

In experiments where oxygen dissolved in perilymph was controlled, it was necessary to substitute a glass tubing system for polyethylene tubing on the inlet side because oxygen significantly diffuses through the walls of most types of plastic tubing (Fig. 3).

To monitor the effect of the perfusion of artificial perilymph and of test substances on the inner ear, auditory electrical responses, the cochlear microphonic 
(A)
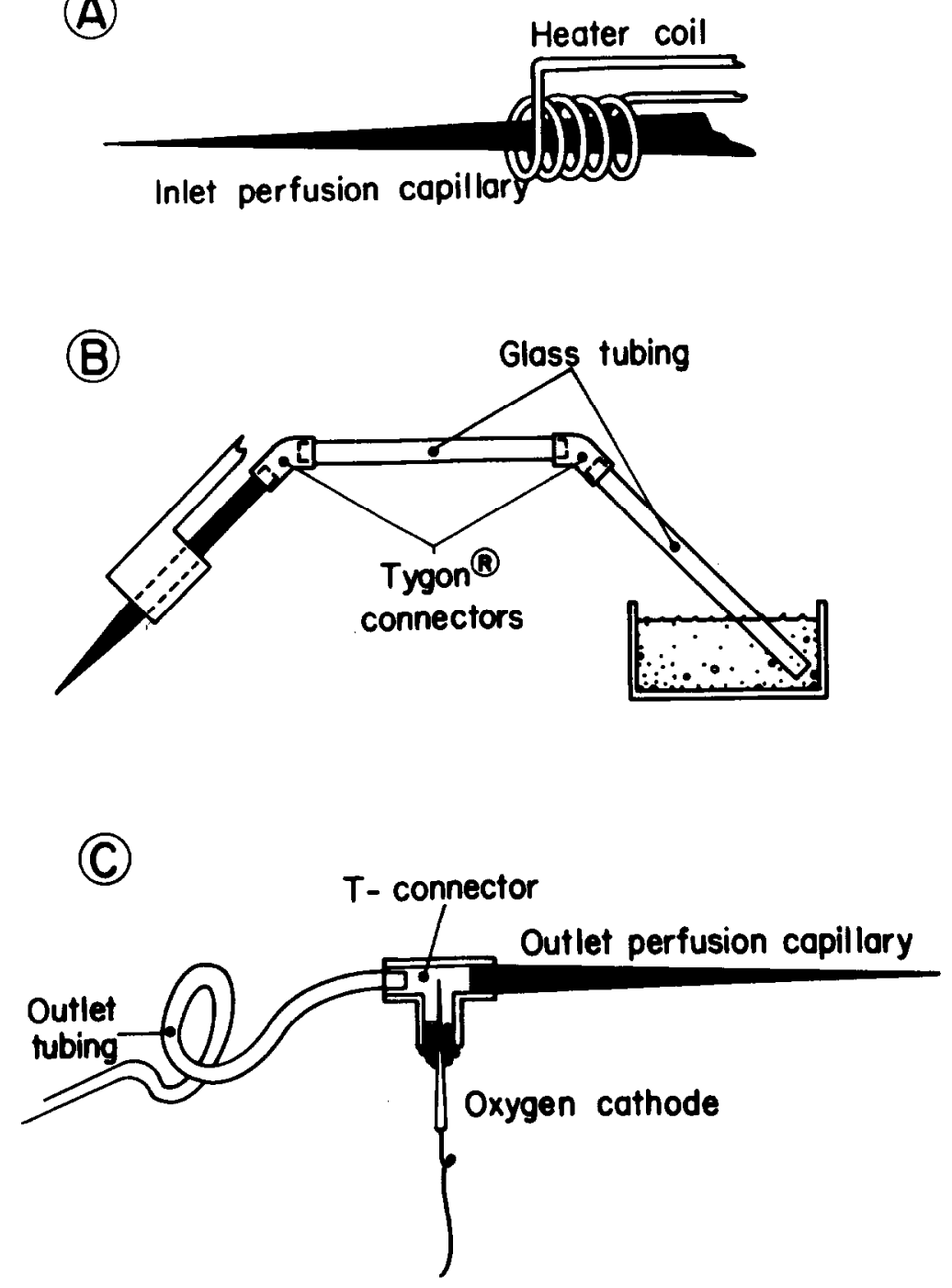

Fig. 3. Details of apparatus used in the perfusion method. (A) A heater coil on the inlet perfusion capillary controlled perfusate and cochlear temperature. (B) Glass tubing with flexible connectors was used to carry perfusate which had an oxygen concentration below atmospheric level. (C) The arrangement of an oxygen-sensitive electrode for determining the oxygen concentration in the outflowing perfusate.

(CM) and occasionally the compound action potential (AP) were recorded. The CM was generated in response to a continuous tone at $1 \mathrm{kHz}$ or $4 \mathrm{kHz}$ while $\mathrm{AP}$ was evoked by a click-type stimulus. A 1-inch condenser microphone served as the closed field sound source and was connected to the external meatus by an acrylic plastic speculum. The electric signals were obtained from a silver wire placed into the 
perfusion supply reservoir, utilizing the supply tubing system as if it were an electrode connecting to the scala tympani of the cochlea. After amplification the signals were measured with a wave analyzer or an oscilloscope and recorded on an oscillograph. The low resistance of the perilymph-filled tubing causes insignificant signal loss for experiments which utilize relative measurements.

The flowing perilymph did not add any obvious noise to the CM but often the drops of outflowing perilymph, which fell from the outlet tubing, introduced transient CM clicks. Sometimes these CM transients were a useful indicator of the flow rate as they were part of the chart recordings.

\section{Control of experimental variables}

\section{Pressure and flow}

The experimental variables of pressure and flow are interrelated in the perfusion method. In the experimental design there is an initial choice of constant flow or constant pressure types of fluid delivery systems. Constant pressure is the better procedure because the inner ear is very pressure sensitive and changes in pressure result in changes in the output of the cochlear potentials which will be used to monitor the condition of the inner ear.

Perfusion schemes which maintain constant flow, on the other hand, accomplish this at the expense of adjustments in pressure. A change in the viscosity of the artificial perilymph, as a result of adding an antibiotic, for example, could result in an altered pressure distribution and altered cochlear electrical output.

The choice of flow rate will govern the initial constant hydrostatic pressure which, in turn, is set by physically lowering the outlet capillary. This establishes a pressure differential that is equal to the height of the perilymph column between the fluid surface of the supply reservoir and the opening of the outlet tube. Choice of flow rate depends on the specific goals of the experiment and the choice is a balance between time required to deliver the perilymph to the ear (from the supply reservoir) and considerations of the required turnover rate of perilymph within the cochlea. In general, the greater the flow rate, the more likely is cochlear damage.

The hydrostatic pressure, which establishes the flow, causes a reduction in the level of the cochlear microphonic (CM) and the compound action potential (AP) for a constant sound intensity. This reduction is relatively greater with higher flow rates and possibly is due to a mechanical pressure biasing of the basilar membrane. Perilymph flow within the chochlea would be expected to result in pressure losses along the perilymphatic channels and particularly at the helicotrema, a constriction at the apex of the cochlea.

Fig. 4 illustrates the typical stability of the CM potential over a $1 \mathrm{~h}$ control perfusion period after initial stabilization. A variability of less than $10 \%$ can be expected for the $\mathrm{CM}$ and usually presents as a decreasing $\mathrm{CM}$ but sometimes as a fluctuation or an increase.

After establishing flow, the large initial shift in CM (resulting from the perfusion 


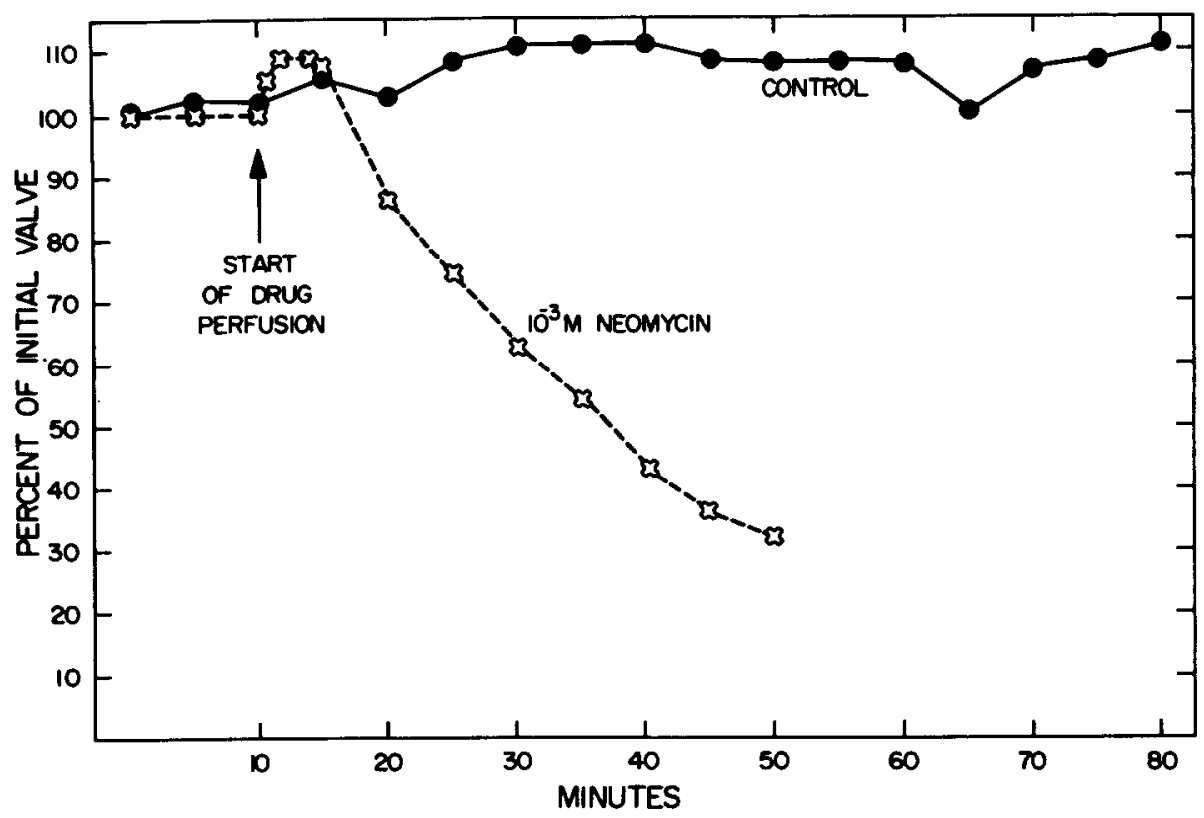

Fig. 4. The percent change of the cochlear microphonic at $4 \mathrm{kHz}$ (initial value $10 \mu \mathrm{V}$ ) during a 70 min control perfusion using artificial perilymph and during a $40 \mathrm{~min}$ perfusion where the artificial perilymph contained $10^{-3} \mathrm{M}$ of the antibiotic neomycin. The flow rate for both experiments was $15 \mu 1 / \mathrm{min}$.

pressure) stabilizes within 5 min and for experimental animals we have established a procedure of allowing a 5-10 min period of stable output before altering the content of the perfusate.

The effect of a perfusion is measured by the percentage amount of CM change or the slope of the change between the start of perfusion and the 30 or $60 \mathrm{~min}$ time point. The greater amount of a change for a given condition will occur by $30 \mathrm{~min}$ so this time point is the better choice. In Fig. 4 , the effect of the ototoxic antibiotic neomycin at a molar concentration of $10^{-3}$ can be compared to the control.

\section{Temperature of the perfusate}

In the perfusion technique there is the possibility of damaging the inner ear by utilizing perfusion fluids that are not adjusted to be at the body temperature. The use of room temperature (approx. $21^{\circ} \mathrm{C}$ ) artificial perilymph is a matter of considerable convenience because delivering heated perilymph through the inlet capillary system is difficult. Simply heating the supply reservoir, for example, does not work because of the slow movement of perilymph through a tube of relatively large surface area completely re-establishes the ambient temperature.

In order to test the temperature variable we first determined the normal cochlear temperature in our experimental condition and discovered that significant cooling of 
the cochlea (to $32^{\circ} \mathrm{C}$ ) occurs during surgery [7]. Perfusion with ambient temperature perilymph then could cause a further decrease of $1-2^{\circ} \mathrm{C}$.

Apparently, the organ of Corti is capable of stable performance under this cooled condition. Nevertheless, the decreased temperature would be expected to reduce the effect of drugs on the inner ear. In fact, this did prove to be true [8].

When a heater was incorporated into the supply capillary system, (illustrated in Figs. 1 and $3 \mathrm{~A}$, as close as possible to the cochlea), the perilymph and cochlear temperature could be controlled and could be measured by a thermocouple on the round window of the cochlea.

The character of the temperature control that can be accomplished is shown in Fig. 5. Four shifts in the temperature registered by a round window thermocouple

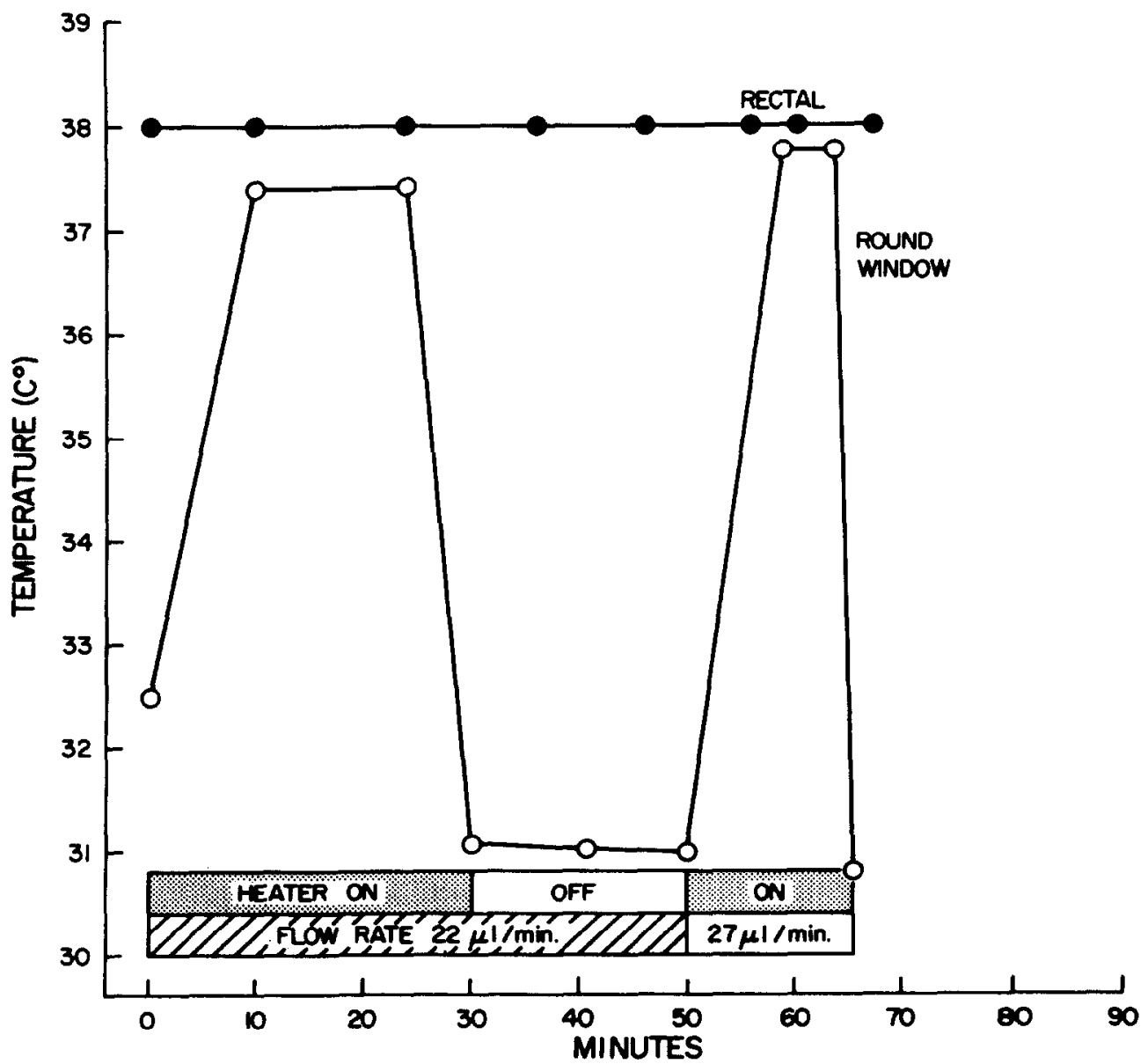

Fig. 5. Alterations in the temperature of the cochlea which could be accomplished by manipulation of the perfusate flow rate and by the application of direct current to the perfusate heater on the inlet capillary. The rectal temperature is held constant by a separate heating pad on the torso of the guinea pig. 
are caused by turning the heater on and off twice. Body temperature measured rectally remained constant. Note that the rate of cochlear heating and cooling is a function of the flow rate. The steady state value of cochlear temperature is also higher with the faster flow (at a given heater current) because the perfusate has less time to cool, between the heater and the cochlea.

As an illustration of the effect of temperature, Fig. 6 shows the effect of the antibiotic, tobramycin, on the CM when tested by perfusion at ambient temperature and at physiologic temperature. The increase in temperature greatly facilitated the drug action but fortunately the temperature effect also proved to be qualitatively a linear effect. Drug concentration differences and drug type differences were preserved at different temperatures [8]. Therefore, depending on the specific goals of the perfusion experiment, it may be appropriate to utilize ambient temperature perfusion.

\section{Effect of perfusate $p H$}

The perfusate $\mathrm{pH}$ is another important parameter of the perfusion method. Jung [2] showed that the $\mathrm{CM}$ is degraded when $\mathrm{pH}$ is shifted from an optimal value near 7.8. This value is greater than the direct intracochlear measurements of endolymph pH (7.3-7.5) made by Misrahy et al. [4]. We reexamined the pH effect on CM and found a narrow range of $\mathrm{pH}$ between approximately 7.3 and 8.2 where the $\mathrm{CM}$ was constant. Outside this range the $\mathrm{CM}$ was rapidly diminished and the effect was irreversible if the perilymph $\mathrm{pH}$ was permitted to become less than 7.0 or greater than 8.2. It should be noted that the outflowing $\mathrm{pH}$ was tested in these experiments in order to ensure that control of intracochlear $\mathrm{pH}$ was being accomplished. At the flow rate of $10-20 \mu \mathrm{l} / \mathrm{min}$, the inner ear did not alter the perfusate $\mathrm{pH}$.

Perfusate $\mathrm{pH}$ is normally adjusted with HEPES buffer and small amounts of $\mathrm{NaOH}$ or $\mathrm{HCl}$ before the perfusate is placed into the supply reservoir. Often there is a slow drift toward more basic levels due to the escape of $\mathrm{CO}_{2}$ from the bicarbonate buffer of the artificial perilymph. Thus, when new samples of perfusate are added to the reservoir, a slight shift in $\mathrm{pH}$ can occur. This in turn can sometimes be detected as small shifts in base line $\mathrm{CM}$ when the perfusate reaches the cochlea. The 'new' $\mathrm{CM}$ level is then taken as the base level.

The important effect of $\mathrm{pH}$ on the inner ear represents a relatively unstudied mechanism for inner ear performance changes with metabolic or blood chemistry alterations.

\section{Manipulation of artificial perilymph oxygen concentration}

The manipulation of the dissolved gasses within the perfusate is a useful tool to study the metabolism of the inner ear. Unfortunately, like temperature, the procedure of manipulating perfusate gas concentration adds considerable complexity to the experiment. The principal difficulty is ensuring that the perfusate does not alter its gas concentrations while in the delivery tubing to the cochlea. Glass tubing was found necessary to accomplish this. Flexibility of the tubing was accomplished with 


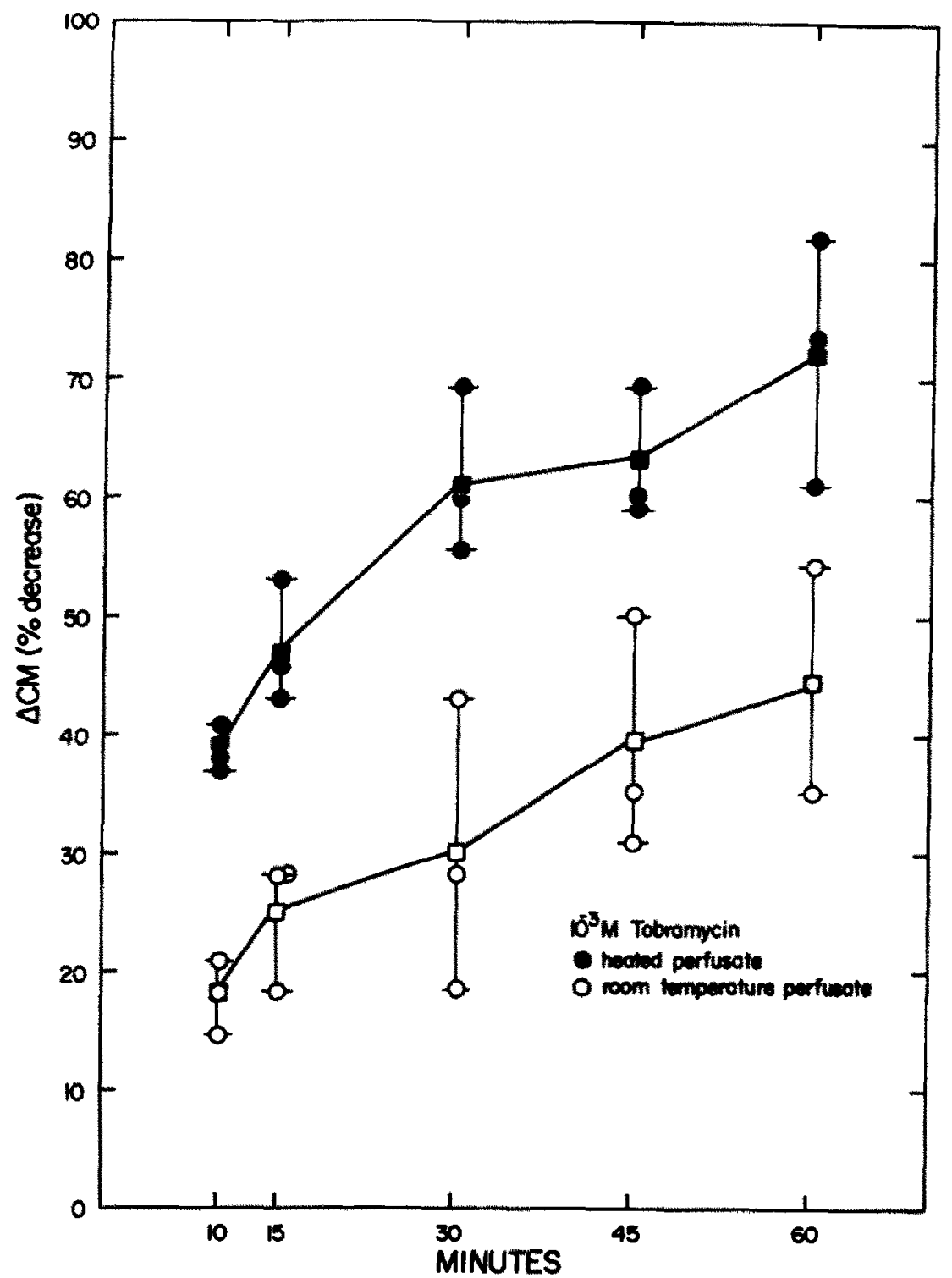

Fig. 6. The temperature dependent effect of the antibiotic, tobramycin $\left(10^{-3} \mathrm{M}\right)$, on the cochlear microphonic potential $(4 \mathrm{kHz}$, initial value $10 \mathrm{\mu V}$ ) when perfused for $60 \mathrm{~min}$. The open symbols represent the use of roon temperature perfusate while filled symbols are for heated perfusate (round window temperature $37^{\circ} \mathrm{C}$ ). Circles are actual data values. Vertical bars represent the range of the data. Squares are average values. Flow rates were $15 \mu \mathrm{l} / \mathrm{min}$.

Tygon $^{\star}$ tubing couplings between straight glass tubing segments (Fig. 3b).

In order to test whether the perfusion technique could control the oxygen level within the perilymphatic spaces of cochlea, an oxygen-sensitive microelectrode was 
incorporated within the outlet tubing (Fig. 3). The oxygen electrode could detect the degree to which the perilymph flowing out of the cochlea was altered when perfusing with deoxygenated perilymph or when perfusing with oxygenated perilymph while turning off the respirator.

The typical results of these experiments are illustrated by Fig. 7. When the respirator was turned off for a $90 \mathrm{~s}$ period, the usual decline/recovery in cochlear microphonic (for a $4 \mathrm{kHz}$ acoustic pure tone) was observed (Fig. 7a). After a delay of 2 to $3 \mathrm{~min}$ the oxygen tension of the perfusate declined and recovered indicating an oxygen utilization during the anoxic period. The delay is caused by the flow time between the cochlea and the measuring electrode. The results of a complementary
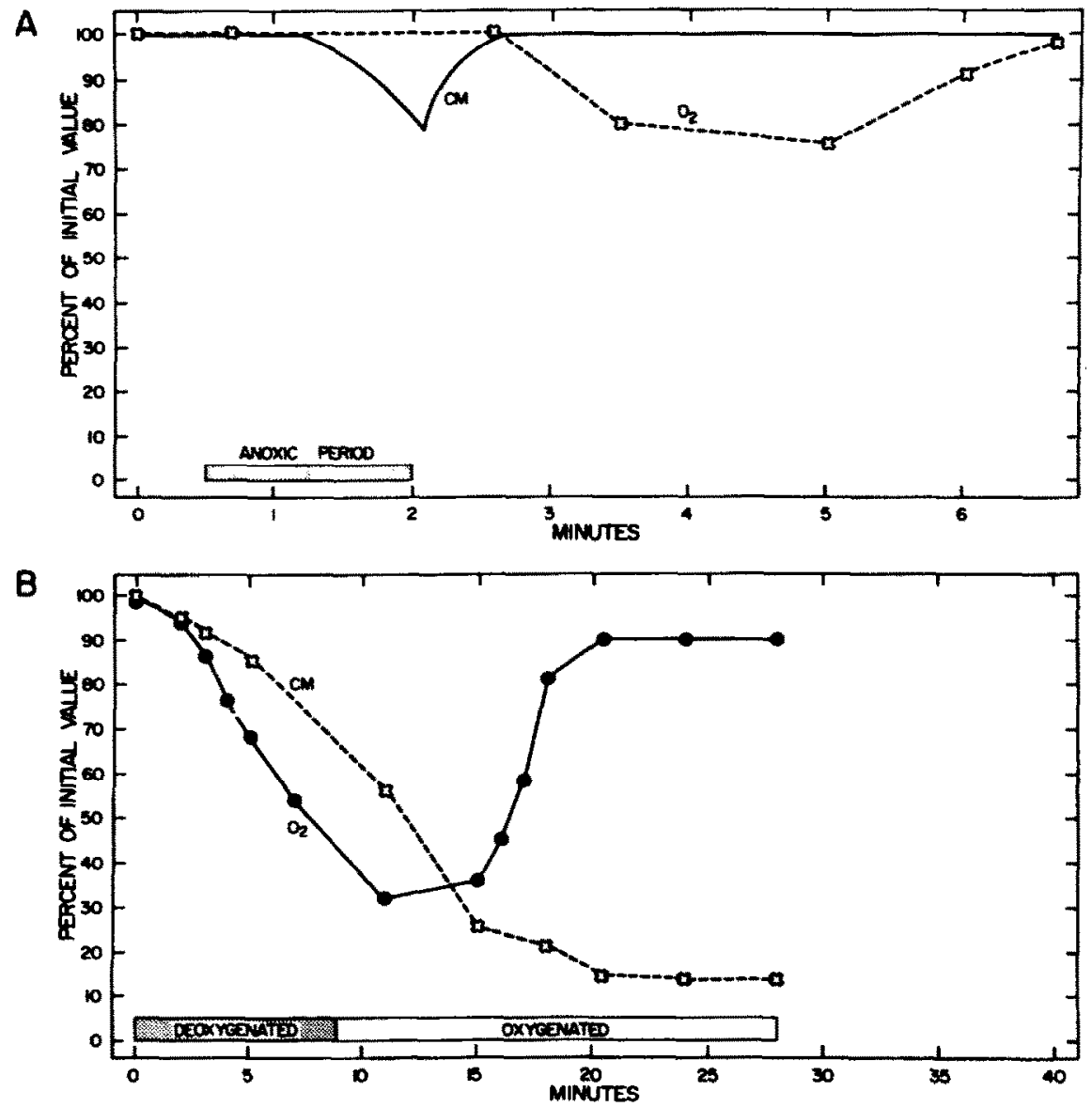

Fig. 7. (A) The effect of a $90 \mathrm{~s}$ whole body anoxia on the cochlear microphonic potential (solid curve) and on the oxygen concentration measured in the outflowing perfusate (broken curve) from tne cochlea. (B) The effect of oxygen depleted perfusate on the cochlear microphonic ( - ) and the oxygen concentration in the outflowing perfusate $(. .$.$) from the cochlea. Flow rates were 11 \mu 1 / \mathrm{min}$. 
experiment are shown in Fig. $7 \mathrm{~b}$ where, instead of a whole body anoxia, the cochlea was perfused with deoxygenated perilymph. The $\mathrm{CM}$ in this case was also depressed but the depression was much greater and became permanent. Okumura [9] observed a similar permanent $\mathrm{CM}$ depression after perfusion of perilymph with $0.25 \%$ glucose-glucoseoxidase and concluded an important oxygen supply path to the hair cells of the cochlea was by way of perilymph.

The results of the two experiments illustrated in Fig. 7 can be interpreted as follows. The first experiment indicates that there is an aerobic metabolism within the cochlea, the need of which is greater than that oxygen capacity of the artificial perilymph flowing at $11 \mu \mathrm{l} / \mathrm{min}$, a rate which exchanges the entire volume of cochlear perilymph in less than $80 \mathrm{~s}$. The second experiment indicates that, at the same flow rate, the blood microcirculation system of the cochlea cannot reoxygenate the oxygen depleted artificial perilymph and the permanent loss of CM is due to the much longer effective period of intracochlear hypoxia.

Although it appears that the loss of $\mathrm{CM}$ is highly correlated with oxygen concentration, it is in fact not possible to infer the causal relationship from this data. The CM stability was also found to be a function of the flow rate parameter. Because of this, the reduction of CM may be related to the buildup of metabolic waste products as well as the depletion of oxygen $[1,6]$.

\section{Summary}

The perilymphatic perfusion method which has been under development and use at this laboratory for four years has reached a state of refinement where both the necessary control studies have been done and a wide ranging usefulness has been demonstrated. In the preceding sections we have detailed the method and shown that pressure and flow can be controlled to result in a stable inner ear response to sound while at the same time the entire fluid volume of perilymphatic space of the cochlea is being cleared between once every minute to once every $3 \mathrm{~s}$.

Perfusion makes it possible to administer drugs into the cochlea with constant concentration conditions. There can be control of $\mathrm{pH}$, temperature and dissolved oxygen gas concentration of the perfusate. All of these variables provide a great flexibility for the application of this method to study inner ear physiology, metabolism, and response to ototoxic agents.

\section{Acknowledgements}

This work was supported by Public Health Service grants NS-05785, NS-11731 and NS-15107, and a Fellowship Award to M.J.LaR., NS-07197.

\section{References}

1 Honnubia, V., Johnstone, B.M. and Butler, R.A. (1965) Maintenance of cochlear potentials during asphyxia. Acta Otolaryngol. 60, 105-111. 
2 Jung, W. (1971) Zur pH-Abhängigkeit des Mikrofonpotentials der Meerschweinchen-Cochlea. Arch. Klin. Exp. Ohren- Nasen- Kehlkoptheilkd. 199, 473-477.

3 Konishi, T. (1979) Effects of local application of ototoxic antibiotics on cochlear potentials in guinea pigs. Acta Otolaryngol. 88, 41-46.

4 Misrahy, G.A., Hildreth, K.M., Clark, L.C. and Shinabarger, E.W. (1958) Measurement of the pH of the endolymph in the cochlea of guinea pigs. Am. J. Physiol. 194, 393-395.

5 Nuttall, A.L., Marques, D.M. and Lawrence, M. (1977) Effects of perilymphatic perfusion with neomycin on the cochlear microphonic potential in the guinea pig. Acta Otolaryngol. 83, 393-400.

6 Nuttall, A.L. and LaRouere, M.J. (1980) Unpublished observations.

7 Nuttall, A.L. and LaRouere, M.J. (1980) Depression of the guinea pig cochlear temperature caused by anesthesia and ventral-approach ear surgery. J. Acoust. Soc. Am. 68, 489-493.

8 Nuttall, A.L. (1981) Perfusion of aminoglycosides in perilymph. In: Aminoglycoside Ototoxicity. Editors: G.J. Matz, S. Lerner, J.E. Hawkins, Jr. Little Brown, Boston.

9 Okumura, H. (1970) Perilymph as a medium of oxygen-supply for the organ of Corti. Arch. Klin. Exp. Ohren- Nasen- Kehlkopfheilkd. 195, 257-265.

10 Tasaki, I. and Fernandez, C. (1952) Modification of cochlear microphonics and action potentials by $\mathrm{KCl}$ solution and by direct currents. J. Neurophysiol. 15, 497-507. 